\title{
A NOTE ON THE DYNAMICS OF AN OSCILLATOR IN THE PRESENCE OF STRONG FRICTION
}

\author{
H. AMANN AND J.I. DIAZ
}

\begin{abstract}
We study the longtime behavior of the solutions of a second order autonomous differential equation, differing from the one of a harmonic oscillator by a nonlinear friction term being only Hölder continuous. In particular, we show that there are two solution curves reaching the rest point in finite time.
\end{abstract}

\section{Introduction}

In this note we study the phase plane flow of the equation

$$
\ddot{x}+\varphi(\dot{x})+x=0,
$$

with the friction function $\varphi$ possessing the following properties:

it is continuous on $\mathbb{R}$ and locally Lipschitz continuous on $\mathbb{R} \backslash\{0\}$,

odd, and positive for positive arguments;

there exist $a, \varepsilon>0$ and $\alpha \in(0,1)$ such that $\varphi(\eta)=a \eta^{\alpha}$ for $0<\eta \leq \varepsilon$;

$\varlimsup_{\eta \rightarrow \infty} \varphi(\eta) / \eta<1 / 2$.

Clearly, if $\varphi$ is defined by

$$
\varphi(\eta):=\operatorname{sign}(\eta)|\eta|^{\alpha}, \quad \eta \in \mathbb{R},
$$

then it satisfies $(0.2)$ whenever $\alpha \in(0,1)$, and the corresponding differential equation is

$$
\ddot{x}+|\dot{x}|^{\alpha-1} \dot{x}+x=0 .
$$

A nonmonotone example is given by

$$
\varphi(\eta):=\left\{\begin{array}{lr}
a \eta^{\alpha}, & 0 \leq \eta \leq \varepsilon, \\
1+1 / \eta, & \varepsilon<\eta<\infty, \\
-\varphi(-\eta), & -\infty<\eta<0,
\end{array}\right.
$$

where $a=\varepsilon^{-1-\alpha}+\varepsilon^{-\alpha}$. This nonlinearity appears in the literature since the pioneering work of Lord Rayleigh (see [4], [5]).

Functions satisfying (0.2) are often used to provide continuous approximations of the Coulomb friction problem

$$
\ddot{x}+\operatorname{sign}(\dot{x})+x \ni 0,
$$

1991 Mathematics Subject Classification. Primary 34C05, 34D05; Secondary 34C15, 34C35.

Key words and phrases. phase plane analysis, stability, asymptotic behavior, Hölder continuous nonlinearity. 
where sign is meant to represent the maximal monotone graph in $\mathbb{R}^{2}$, given by -1 for $\eta<0$, by $[-1,1]$ for $\eta=0$, and by 1 for $\eta>0$.

It is easy to see (e.g., [4]) that, in the Coulomb friction problem, every point in the phase plane reaches a critical point $(\xi, 0)$ with $\xi \in[-1,1]$ in finite time by the flow induced by (0.6). In the case of equation (0.1) the origin is the only critical point of the phase flow, that is, the flow generated by

$$
\dot{x}=y, \quad \dot{y}=-x-\varphi(y) .
$$

From (0.2) and (0.7) we deduce that

$$
\left(x^{2}+y^{2}\right)^{\cdot}=-2 \varphi(y) y<0, \quad y \neq 0 .
$$

Thus, given any $R>0$, the disc $\overline{\mathbb{B}}_{R}:=\left\{(x, y) \in \mathbb{R}^{2} ; x^{2}+y^{2} \leq R^{2}\right\}$ is positively invariant. Hence the origin is globally asymptotically stable, that is, it is stable and every point $(x, y) \in \mathbb{R}^{2} \backslash\{(0,0)\}$ is transported to the origin by the flow. (Henceforth we simply say that 'the orbit through $(x, y)$ reaches the origin', etc. )

Since, as mentioned above, equation (0.1) can be considered to be an approximation of the Coulomb friction problem, there arises the question whether the critical point is reached in finite time in this case as well. In [2] and [3] it is shown that for problem (0.4) there exist at least two orbits reaching the origin in finite time and there is an albeit small subset $M$ of $\mathbb{R}^{2} \backslash\{(0,0)\}$ such that each orbit meeting $M$ needs infinite time to reach the rest state. The proofs are based on asymptotic methods and comparison theorems and make use of the explicit form (0.3) of $\varphi$.

In this paper we show by completely different and elementary techniques from the theory of ordinary differential equations that, given assumptions (0.2), problem (0.7) possesses also at least two orbits converging to the rest state in finite time. Furthermore, we give a rather detailed analysis of the flow in the neighborhood of the critical point, showing that most orbits reach it in infinite time only. In fact, on the basis of our results we conjecture that there are precisely two orbits converging to zero in finite time.

\section{The semiflow on the upper half-plane}

First we observe that the oddness of $\varphi$ implies that the phase portrait of (0.7) is invariant under the reflection $(x, y) \mapsto(-x,-y)$. Thus it suffices to study the semiflow on the closure of the upper half-plane $\mathbb{H}:=\left\{(x, y) \in \mathbb{R}^{2} ; y>0\right\}$, induced by restriction from the flow generated by (0.7). Consequently, in the rest of this section all assertions on invariant regions etc. pertain to this semiflow. (We refer to [1] for the elementary facts about semiflows and invariant regions which we use without further mention.)

From $(0.7)$ we see that every orbit meets the real axis off zero vertically. For $0<s \leq 1$ we denote by $\Gamma_{s}$ the curve

$$
\Gamma_{s}:=\{(x, y) \in \mathbb{H} ;-x=s \varphi(y)\},
$$

oriented so that $y$ is decreasing. Then every orbit in $\mathbb{H}$ meets $\Gamma:=\Gamma_{1}$ horizontally, and the direction field has a positive $x$-component and a negative $y$-component on the right of $\Gamma$. Thus no orbit approaches the origin through the first quadrant. Furthermore, given any $\eta>0$,

$$
\{(x, y) \in \mathbb{H} ;-x \leq \varphi(y), y \leq \eta\}
$$


is positively invariant.

We denote by $C(x, y)$ the solution curve of $(0.7)$ passing through $(x, y) \in \mathbb{H}$ and being oriented in positive time direction.

1.1 Lemma There exists $\eta^{*}>0$ such that $C(x, y)$ meets the positive $y$-axis whenever $(x, y) \in \Gamma_{s}$ for some $s \in(0,1]$ and satisfies $y \geq \eta^{*}$.

Proof. Suppose that $\left(x_{0}, y_{0}\right) \in \Gamma_{s}$ and $C\left(x_{0}, y_{0}\right)$ stays for positive time in

$$
\mathbb{H}^{-}:=\{(x, y) \in \mathbb{H} ; x<0\} .
$$

From (0.2) we deduce the existence of $\alpha<1 / 2$ and $\bar{\eta}>0$ such that

$$
\varphi(\eta) \leq \alpha \eta, \quad \eta \geq \bar{\eta}
$$

Fix $\beta \in(1 / 2,1-\alpha)$ and suppose that

$$
y_{0} \geq \bar{\eta} / \beta=: \eta^{*}
$$

Let $t^{*}$ be the time at which $C\left(x_{0}, y_{0}\right)$ reaches the $\eta$-level, that is, $y\left(t^{*}\right)=\beta y_{0}$. Then we deduce from the second equation in $(0.7),(1.1)$, and (1.2) that

$$
t^{*}=\int_{\beta y_{0}}^{y_{0}} \frac{d y}{x+\varphi(y)} \geq \int_{\beta y_{0}}^{y_{0}} \frac{d y}{\varphi(y)} \geq \frac{1-\beta}{\alpha},
$$

thanks to $x(t)<0$ for $0 \leq t \leq t^{*}$. By integrating the first equation of $(0.7)$ we obtain

$$
x\left(t^{*}\right)-x_{0}=\int_{0}^{t^{*}} y d t \geq \beta y_{0} t^{*} .
$$

Thus, using $x_{0}=-s \varphi\left(y_{0}\right)$ and (1.1), we deduce from (1.3) that

$$
x\left(t^{*}\right) \geq x_{0}+\frac{\beta(1-\beta)}{\alpha} y_{0} \geq \varphi\left(y_{0}\right)\left(\frac{\beta(1-\beta)}{\alpha^{2}}-s\right)>0,
$$

thanks to $\alpha<1 / 2<\beta<1-\alpha$. But this contradicts the assumption that $C\left(x_{0}, y_{0}\right)$ stays in $\mathbb{H}^{-}$and thus proves the assertion.

For $0<s<1$ and $(x, y) \in \Gamma_{s}$ the positive unit tangent vectors to $C(x, y)$ and $\Gamma_{s}$ at $(x, y)$ are given by

$$
(y,(1-s) \varphi(y)) / r(s, y), \quad\left(s \varphi^{\prime}(y),-1\right) / \rho(s, y),
$$

respectively, where

$$
r(s, y):=\sqrt{y^{2}+(1-s)^{2}[\varphi(y)]^{2}}, \quad \rho(s, y):=\sqrt{1+s^{2}\left[\varphi^{\prime}(y)\right]^{2}},
$$

provided $\varphi$ is differentiable at $y$. In this case these vectors are equal iff

$$
y=\psi(s, y) s \varphi^{\prime}(y), \quad(1-s) \varphi(y)=\psi(s, y)
$$

where $\psi(s, y):=r(s, y) / \rho(s, y)$, that is, iff

$$
y=s(1-s) \varphi(y) \varphi^{\prime}(y) .
$$

Note that (0.2) implies

$$
\varphi(y)=a y^{\alpha}, \quad \varphi^{\prime}(y)=\alpha \varphi(y) / y, \quad 0<y \leq \varepsilon .
$$

Hence we deduce from (1.5), setting

$$
\beta:=1 / 2(1-\alpha), \quad \lambda(s):=\alpha a^{2} s(1-s),
$$

that

$$
y=k_{2}(s):=[\lambda(s)]^{\beta},
$$


provided $0<y \leq \varepsilon$. Furthermore, $(x, y) \in \Gamma_{s}$ implies in this case

$$
x=k_{1}(s):=-s \varphi\left(k_{2}(s)\right)=-s a[\lambda(s)]^{\alpha \beta} .
$$

Set

$$
s_{\varepsilon}:= \begin{cases}\frac{1}{2}-\frac{1}{2} \sqrt{1-\frac{4}{\alpha a^{2}} \varepsilon^{2(1-\alpha)}} & \text { if } \varepsilon<\left(\frac{\alpha a^{2}}{4}\right)^{1 / 2(1-\alpha)} \\ \frac{1}{2} & \text { otherwise. }\end{cases}
$$

Define the oriented curve $K:=K_{\varepsilon}$ in $\mathbb{H}^{-}$by means of the parametrization

$$
\left(0, s_{\varepsilon}\right] \rightarrow \mathbb{H}^{-}, \quad s \mapsto k(s):=\left(k_{1}(s), k_{2}(s)\right) .
$$

Then, by the preceding considerations,

$$
k(s) \in \Gamma_{s}, \quad 0<s \leq s_{\varepsilon},
$$

and the positive unit tangent vectors to $C(x, y)$ and $\Gamma_{s}$ at $(x, y)=k(s)$ coincide for $0<s \leq s_{\varepsilon}$.

1.2 Lemma The region $M$ in $\mathbb{H}^{-}$, bounded by $\left\{\left(k_{1}\left(s_{\varepsilon}\right), \eta\right) ; 0<\eta \leq k_{2}\left(s_{\varepsilon}\right)\right\}$ and $K$, is positively invariant.

Proof. It is clear that the direction field of (0.7) is inward pointing along the vertical boundary of $M$.

From (1.6) and (1.8) we deduce that

$$
\dot{k}_{1}(s)=-\varphi\left(k_{2}(s)\right)\left[1+\alpha s \dot{k}_{2}(s) / k_{1}(s)\right] .
$$

Note that (1.7) implies

$$
\frac{\dot{k}_{2}(s)}{k_{2}(s)}=\beta \frac{\dot{\lambda}(s)}{\lambda(s)}=\beta\left(\frac{1}{s}-\frac{1}{1-s}\right) .
$$

Hence

$$
\dot{k}_{1}(s)=k_{1}(s)\left[\frac{1}{s}+\alpha \beta \frac{\dot{\lambda}(s)}{\lambda(s)}\right] .
$$

The positive normal to $K$ at $k(s)$ has the direction of $\left(-\dot{k}_{2}(s), \dot{k}_{1}(s)\right)$. Consequently, the inner product of the positive unit normal to $K$ and the positive unit tangent vector to $C(x, y)$ at $(x, y)=k(s)$ is a positive multiple of

$$
\begin{aligned}
& \left(-\dot{k}_{2}(s), \dot{k}_{1}(s)\right) \cdot\left(k_{2}(s),-k_{1}(s)-\varphi\left(k_{2}(s)\right)\right) \\
& \quad=-\left[k_{2}(s)\right]^{2} \beta \frac{\dot{\lambda}(s)}{\lambda(s)}+\left[\varphi\left(k_{2}(s)\right)\right]^{2} s(1-s)\left(\frac{1}{s}+\alpha \beta \frac{\dot{\lambda}(s)}{\lambda(s)}\right) \\
& \quad=-\alpha a^{2} \beta[\lambda(s)]^{2 \beta-1}(1-2 s)+a^{2} \lambda^{2 \alpha \beta}(1-s+\alpha \beta[1-s-s]) \\
& \quad=a^{2}[\lambda(s)]^{2 \alpha \beta}(1-s)>0 .
\end{aligned}
$$

Thus the direction field of (0.7) is inward pointing along $K$ as well. This implies the assertion.

1.3 Proposition There exists a positive semiorbit in $\mathbb{H}^{-}$reaching the origin in finite time. 
Proof. Let $s \in\left(0, s_{\varepsilon}\right]$ be fixed and put

$$
S:=\left\{(x, y) \in \Gamma_{s} ; C(x, y) \text { meets the } y \text {-axis in } \mathbb{H}\right\} .
$$

Then $S \neq \emptyset$ by Lemma 1.1. Put

$$
\bar{y}:=\inf \left\{y \in \mathbb{R}^{+} ;(x, y) \in S\right\} .
$$

If $(x, y) \in \Gamma_{s}$ and $y \leq k_{2}(s)$ then it follows from Lemma 1.2 that $(x, y) \notin S$. Hence $\bar{y} \geq k_{2}(s)>0$.

For $(x, y) \in S$ we denote by $t(y)$ the time at which $C(x, y)$ meets the positive $y$-axis first, at $\left(0, y_{0}\right)$ say. Then, since $x(t) \geq-s \varphi(y(t))$ for $0 \leq t \leq t(y)$, it follows by integrating the first equation in (0.7) that

$$
t(y)=\int_{0}^{t(y)} d t=\int_{y}^{y_{0}} \frac{d y}{-x-\varphi(y)} \leq \frac{1}{1-s} \int_{0}^{y} \frac{d y}{\varphi(y)} .
$$

Thus fix $\widehat{y} \in S$. Then

$$
t(y) \leq \frac{1}{1-s} \int_{0}^{\widehat{y}} \frac{d y}{\varphi(y)}=: T<\infty, \quad \bar{y} \leq y \leq \widehat{y} .
$$

Hence we deduce from the continuous dependence of the flow on the initial value and the compactness of the interval $[0, T]$ that $\bar{y} \in S$. This proves the assertion since $t(\bar{y}) \leq T$.

In the following picture we depict $\mathbb{H}^{-}$in the neighborhood of the origin together with $K, \Gamma$, and $\Gamma_{s}$ for three values of $s$, as well as seven orbits for problem (0.5) with $\alpha=0.4$ and $\varepsilon=1.2$.

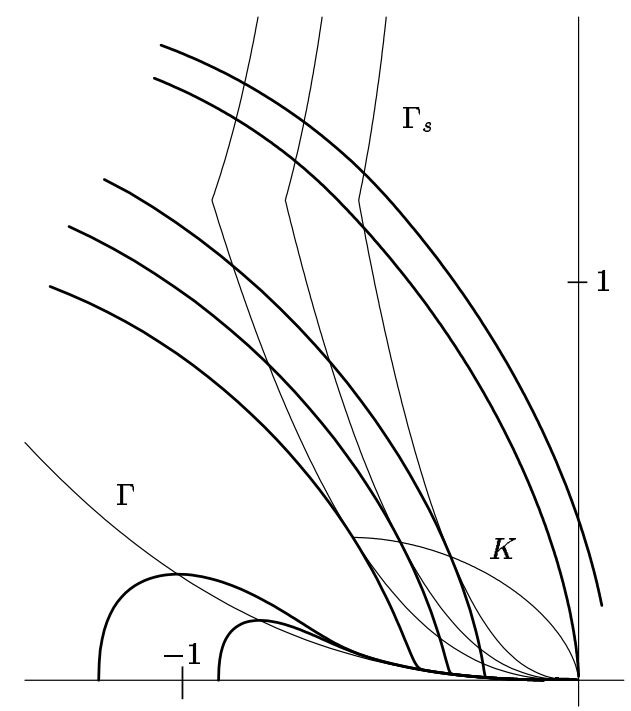

Finally, we prove that there are orbits needing infinite times to reach the origin.

1.4 Proposition Every orbit meeting $M$ needs infinite time to reach the origin.

Proof. Choose $s \in\left(0, s_{\varepsilon}\right]$ and suppose that $(\xi, \eta) \in \Gamma_{s} \cap M$. From (1.4) we deduce that the inner product of the unit tangent vector to $C(\xi, \eta)$ with the negative unit 
normal to $\Gamma_{s}$ at $(\xi, \eta)$ is a positive multiple of

$$
\begin{aligned}
\gamma(s, \eta) & :=s(1-s) \varphi(\eta) \varphi^{\prime}(\eta)-\eta=\eta\left[\alpha a^{2} s(1-s) \eta^{2(\alpha-1)}-1\right] \\
& =\eta\left[\lambda(s) \eta^{2(\alpha-1)}-1\right] .
\end{aligned}
$$

From $0<\eta \leq k_{2}(s)=[\lambda(s)]^{\beta}$ it follows that $\gamma(s, \eta) \geq \gamma\left(s, k_{2}(s)\right)=0$. This shows that, given any $s$, the subregion of $M$ lying below $\Gamma_{s}$ is positively invariant.

For $(x, y) \in M$ there is a unique $s \in\left(0, s_{\varepsilon}\right]$ such that $\Gamma_{s}$ meets $K$ in $\left(x, y_{s}\right)$, where $y_{s}$ satisfies $s \varphi\left(y_{s}\right)=-x$. Thus the positive orbit starting at time zero in $(x, y)$ lies below $\Gamma_{s}$, that is, $s \varphi(y(t)) \leq-x(t)$ for $0 \leq t<t^{+}$, where $t^{+}$is the positive escape time of $(x, y)$. Consequently, by integrating the first equation of $(0.7)$, we see that

$$
t^{+}=\int_{0}^{t^{+}} d t=\int_{x}^{0} \frac{d x}{y} \geq \int_{x}^{0} \frac{d x}{(-x / a)^{1 / \alpha}}=\int_{0}^{-x / a} \frac{d \xi}{\xi^{1 / \alpha}}=\infty,
$$

thanks to $1 / \alpha>1$.

\section{The final result}

After the preceding preparations we can now prove the main theorem of this paper. It gives a rather precise picture of the flow on $\mathbb{R}^{2}$ generated by $(0.7)$.

2.1 Theorem The origin is the only critical point of the phase flow of (0.1), and it is globally asymptotically stable. More precisely, given any $R>0$, the disc $\overline{\mathbb{B}}_{R}$ is positively invariant and every orbit converges to the rest state. The phase portrait is symmetric with respect to the origin.

There exist real numbers satisfying $0<\bar{x} \leq \widehat{x}$ with the following properties:

(i) Every orbit meets $[-\widehat{x}, \widehat{x}] \times\{0\}$.

(ii) If an orbit meets $[-\widehat{x}, 0) \times\{0\}$, resp. $(0, \widehat{x}] \times\{0\}$, then it stays in $\mathbb{H}^{-}$, resp.

$$
(-\mathbb{H})^{+}:=\left\{(x, y) \in \mathbb{R}^{2} ; x>0, y<0\right\} .
$$

If an orbit meets $(-\infty,-\widehat{x}) \times\{0\}$, resp. $(\widehat{x}, \infty) \times\{0\}$, then it leaves $\mathbb{H}^{-}$, resp. $(-\mathbb{H})^{+}$, after finite time.

(iii) The orbits through $(-\widehat{x}, 0)$ and $(0, \widehat{x})$ reach the rest point in finite time.

(iv) Every orbit meeting $[(-\bar{x}, 0) \cup(0, \bar{x})] \times\{0\}$ needs infinite time to reach the critical point.

Proof. The first part of the assertion has already been observed in the Introduction and at the beginning of Section 1.

By Proposition 1.3 and its proof there exists $\left(\widehat{x}_{0}, \widehat{y}_{0}\right) \in \mathbb{H}^{-}$such that the positive semiorbit starting in $\left(\widehat{x}_{0}, \widehat{y}_{0}\right)$ stays in $\mathbb{H}^{-}$, converges to $(0,0)$ in finite time, and every positive semiorbit through $\left(\widehat{x}_{0}, \eta\right)$ with $\eta>\widehat{y}_{0}$ leaves $\mathbb{H}^{-}$by intersecting the positive $y$-axis away from zero. Let $\gamma^{-}\left(\widehat{x}_{0}, \widehat{y}_{0}\right)$ be the negative semiorbit starting at time zero at $\left(\widehat{x}_{0}, \widehat{y}_{0}\right)$. Then it is easily seen that there exists $\widehat{x}>0$ such that $\gamma^{-}\left(\widehat{x}_{0}, \widehat{y}_{0}\right)$ meets $(-\infty, 0) \times\{0\}$ for the first time in $(-\widehat{x}, 0)$. Thus assertions (i)-(iii) are clear. Assertion (iv) is now an easy consequence of what has just been shown and of Proposition 1.4.

In the next picture a portion of the phase plane of problem (0.1) is depicted where $\varphi$ is given by (0.5) with $\alpha=0.4$ and $\varepsilon=1.2$. 


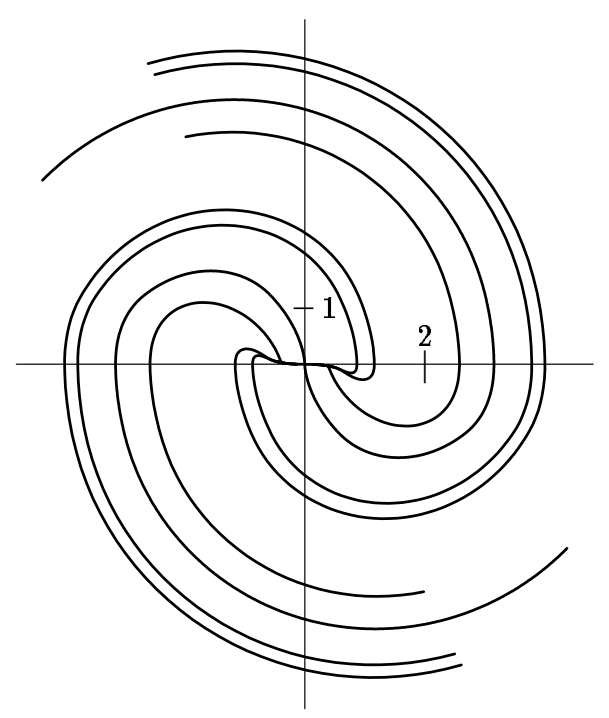

We conjecture, but do not know, that $\bar{x}=\widehat{x}$ in which case there are precisely two orbits reaching the origin in finite time.

\section{References}

[1] H. Amann. Ordinary differential equations, volume 13 of de Gruyter Studies in Mathematics. Walter de Gruyter \& Co., Berlin, 1990. An introduction to nonlinear analysis, Translated from the German by G. Metzen.

[2] J.I. Díaz, A. Liñán. On the asymptotic behavior for a damped oscillator under a sublinear friction. RACSAM Rev. R. Acad. Cienc. Exactas Fis. Nat. Ser. A Mat., 95(1) (2001), 155160.

[3] _ On the asymptotic behavior of solutions of a damped oscillator under a sublinear friction term: from the exceptional to the generic behaviors. In Partial differential equations, volume 229 of Lecture Notes in Pure and Appl. Math., pages 163-170. Dekker, New York, 2002.

[4] D.W. Jordan, P. Smith. Nonlinear ordinary differential equations. Oxford Applied Mathematics and Computing Science Series. The Clarendon Press Oxford University Press, New York, second edition, 1987.

[5] J.W. Rayleigh, B. Strutt. The Theory of Sound. Dover Publications, New York, N. Y., 1945. $2 \mathrm{~d}$ ed.

InStitut für MAthematik, Universität Zürich, WinterthuRERstr. 190, CH-8057 Zürich, SWITZERLAND

E-mail address: amann@math.unizh.ch

Universidad Complutense de Madrid, Ciudad Universitaria, 28040 Madrid, Spain

E-mail address: ji_diaz@mat.ucm.es 